\title{
Normal Pressure Hydrocephalus as an Unusual Presentation of Supratentorial Extraventricular Space-Occupying Processes: Report on Two Cases
}

\author{
E. Naydenov ${ }^{a} \quad$ V. Bussarsky ${ }^{a} \quad$ K. Minkin ${ }^{a} \quad$ A. Bussarsky \\ S. Nachev ${ }^{b}$ L. Traykov ${ }^{c}$ \\ ${ }^{a}$ Department of Neurosurgery and ${ }^{b}$ Laboratory of Neuropathology, University \\ Hospital 'St. Ivan Rilski', 'Department of Neurology, University Hospital \\ 'Alexandrovska', Sofia, Bulgaria
}

\section{Key Words}

Normal pressure hydrocephalus · Meningioma - Glioblastoma multiforme

\begin{abstract}
Normal pressure hydrocephalus (NPH) is a clinical and radiographic syndrome characterized by ventriculomegaly, abnormal gait, urinary incontinence, and dementia. The condition may occur due to a variety of secondary causes but may be idiopathic in approximately $50 \%$ of patients. Secondary causes may include head injury, subarachnoid hemorrhage, meningitis, and central nervous system tumor. Here, we describe two extremely rare cases of supratentorial extraventricular space-occupying processes: meningioma and glioblastoma multiforme, which initially presented with NPH.
\end{abstract}

\section{Introduction}

Normal pressure hydrocephalus (NPH) can be idiopathic or secondary. The latter may occur in the setting of head trauma, subarachnoid hemorrhage (SAH), meningitis, and central nervous system (CNS) tumor [1,2]. The present report describes two unusual cases of supratentorial extraventricular space-occupying processes: meningioma and glioblastoma multiforme (GBM), which initially presented with NPH. 


\section{Case Reports}

\section{Case 1}

A 58-year-old female patient was referred to our clinic with a 6-month history of progressive gait disturbance and urinary incontinence. The neurological exam showed the full clinical picture of NPH syndrome, including remarkable dementia which had been completely ignored by the patient and relatives. The computed tomography (CT) scan demonstrated marked ventriculomegaly as well as a well-delineated left-side temporoparietal lesion, consistent with a convexity meningioma (fig. $1 \mathrm{a}, \mathrm{b}$ ). The ophthalmological examination showed no abnormalities. However, lumbar tap was not performed because of the presence of the intracranial space-occupying process. The patient was operated on in March 2010, with total removal of the tumor. Histological examination revealed World

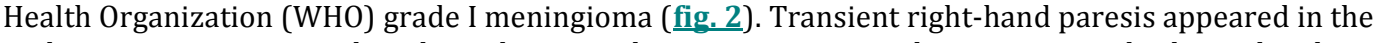
early postoperative period, with no change in the NPH symptoms. The patient was discharged and scheduled for ventriculoperitoneal shunt placement. However, her family refused any further surgical interventions. Surprisingly, 1 month later, the patient presented with significant improvement of the gait difficulties and urinary incontinence. The control CT examination showed no data for residual tumor as well as a slight decrease of the ventricular size (fig. 1c). No additional dynamics of the patient's neurological status were noted until January 2011.

\section{Case 2}

A 68-year-old male patient was referred to the clinic with an 18-month history of involuntary left arm movements and subsequent development of progressive gait disturbances and urinary incontinence. The neuroimaging studies made before the admission demonstrated communicating hydrocephalus without any clinically significant lesions within the brain parenchyma (fig. $3 a, b)$. The ophthalmological examination was normal. Just prior to the hospitalization, the patient started to complain of progressive left arm weakness. The control CT scan revealed a heterodense tumor situated in the right frontotemporal region with compression and dislocation of the adjacent brain structures to the left (fig. 3c). Partial excision of the lesion was performed in June 2010, using frameless stereotaxy technique. Histologically, the tumor demonstrated typical features of GBM (WHO grade IV glioma; fig 4 ). No additional deficit was observed after the intervention. The patient was discharged and scheduled for adjuvant chemoradiation. In spite of the treatment, he died 3 months later from the consequences of GBM.

\section{Discussion}

$\mathrm{NPH}$ is a clinical and radiographic syndrome characterized by ventriculomegaly, gait disturbances, urinary incontinence, and mental decline. The condition may occur due to a variety of secondary causes including head injury, SAH, meningitis, and CNS tumor [1, 2]. A number of possible mechanisms have been suggested to explain the pathogenesis of adult hydrocephalus that occurs in association with intracranial space-occupying processes [3-8].

The blockage of the CSF outflow through the ventricular system is one of the most common pathways for the development of secondary hydrocephalus in adults. The majorities of cases demonstrate rapidly progressing symptoms of increased intracranial pressure and necessitate urgent intervention [3]. However, in some instances, patients may also develop NPH, as described by Lobato et al. [4] and Sayama et al. [5]. Obviously, this mechanism is not relevant to our cases, as both tumors were extraventricular.

According to many authors, the hyperproteinorrachia, which can be observed in some patients with CNS tumors, may cause NPH by impairing CSF reabsorption at the level of the arachnoid granulations [6]. However, in our cases, this mechanism can only be suspected, because no lumbar puncture was performed before surgery. 
The dissemination of CNS tumor into the subarachnoid space may also lead to NPH. Interestingly, the systematic review of the literature revealed only one such case: a 69year-old female patient with leptomeningeal gliomatosis, who initially presented with confusion, progressive memory decline, and urinary incontinence. The repeated CT scan demonstrated slight dilatation of the lateral ventricles, which together with the authors' clinical description supports the diagnosis of NPH, although the latter was not specifically proposed [7]. Even possible in our case with GBM, this mechanism remains unproved, because no autopsy was done.

The intracranial tumors may also be associated with recurrent SAHs. The latter may provoke adult hydrocephalus on the basis of a decreased rate of CSF absorption at the arachnoid villi. For example, Chang et al. [8] described a 51-year-old male patient with spinal leptomeningeal metastases from a giant-cell GBM, who consequently developed $\mathrm{SAH}$ and NPH. In our cases, however, this mechanism is probably of no significance, because in neither of them subarachnoid bleeding was observed.

\section{Conclusion}

The association of supratentorial extraventricular space-occupying processes with $\mathrm{NPH}$ is rather uncommon. The exact mechanism of this phenomenon is unclear and requires further investigation.

\section{Acknowledgement}

The authors wish to thank the Stay Foundation (www.stay.eu.com), Sofia, Bulgaria, for supporting this research project.
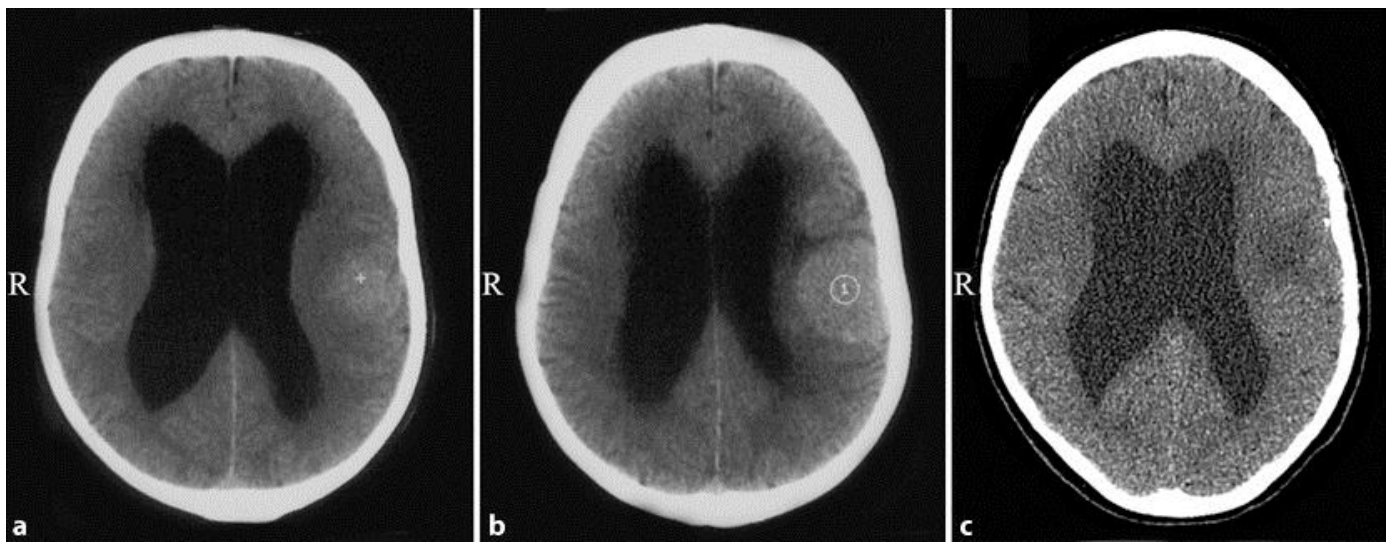

Fig. 1. Neuroimaging of case 1. CT scans before the surgical intervention (a, b) and 1 month later (c). 


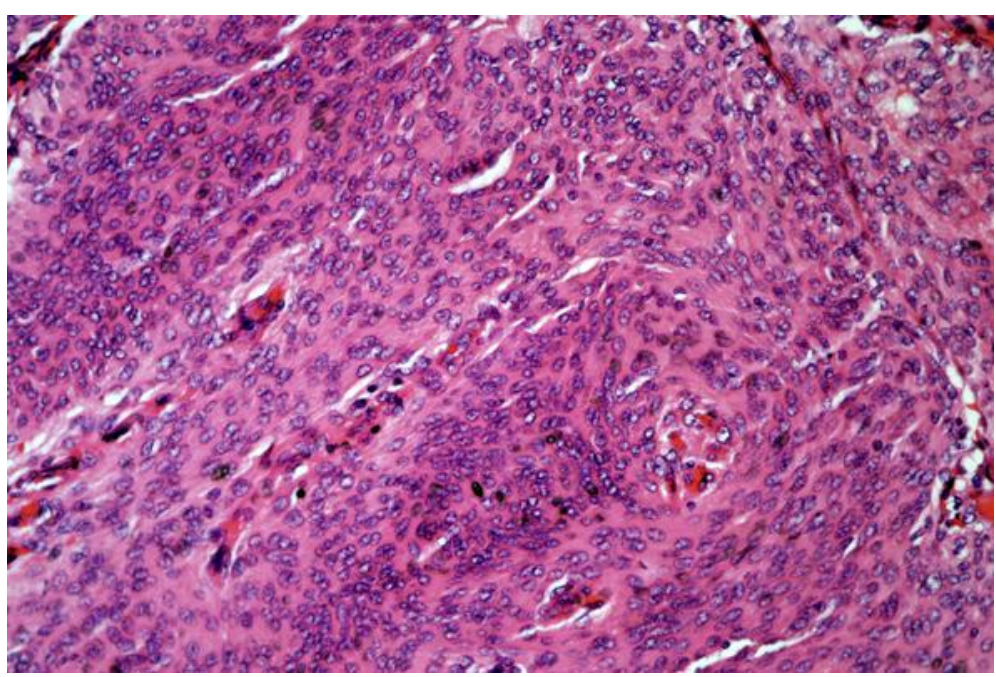

Fig. 2. Histological findings of case 1. The tumor is composed of uniform cells with syncytial growth in sheets and lobules surrounded by thin stroma (WHO grade I meningothelial meningioma). HE, $\times 200$.
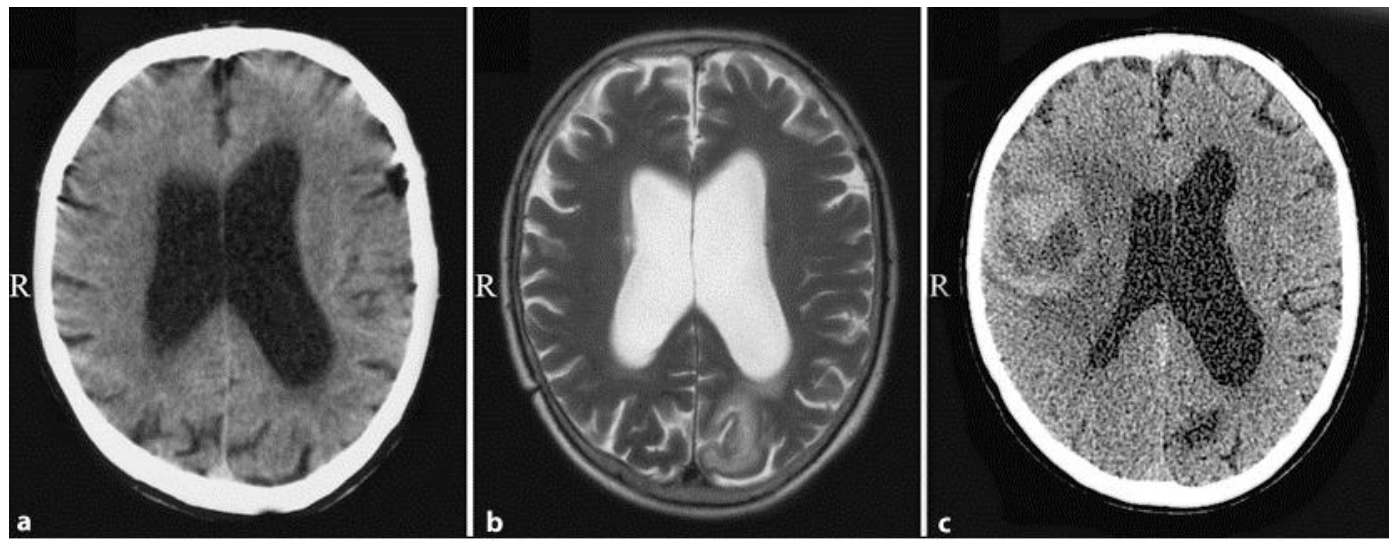

Fig. 3. Neuroimaging of case 2. CT and MRI scans 2 months before the admission (a, $\mathbf{b})$, and CT scan just prior to surgical intervention (c). 


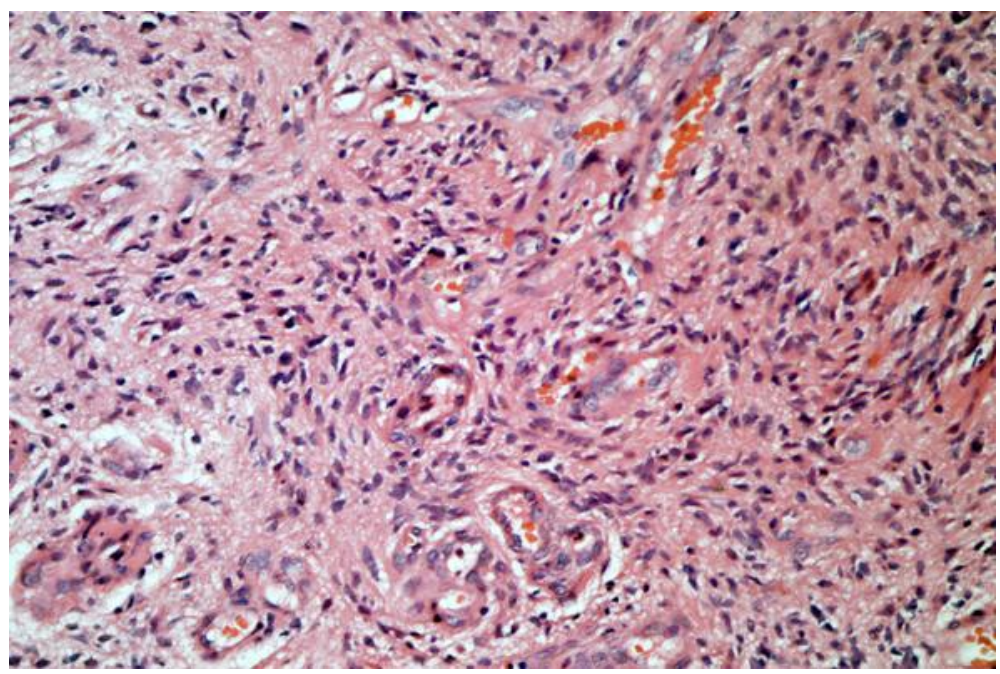

Fig. 4. Histological findings of case 2. The tumor shows prominent cellular polymorphism, microvascular proliferation, and pseudopalisading necroses (WHO grade IV glioma, GBM). HE, $\times 200$.

\section{References}

1 Finney GR: Normal pressure hydrocephalus. Int Rev Neurobiol 2009;84:263-281.

-2 Tisell M, Hellström P, Ahl-Börjesson G, Barrows G, Blomsterwall E, Tullberg M, Wikkelsö C: Long-term outcome in 109 adult patients operated on for hydrocephalus. Br J Neurosurg 2006;20:214-221.

-3 Yoshida S, Takahashi H: Cerebellar metastases in patients with cancer. Surg Neurol 2009;71:184-187.

4 Lobato RD, Lamas E, Cordobés F, Muñoz MJ, Roger R: Chronic adult hydrocephalus due to uncommon causes. Acta Neurochir (Wien) 1980;55:85-97.

5 Sayama I, Yasui N, Fukasawa H, Nemoto M, Ohta H: A case of pineocytoma presenting with symptoms like normal pressure hydrocephalus. No Shinkei Geka 1986;14:789-794.

6 Bloch J, Vernet O, Aubé M, Villemure JG: Non-obstructive hydrocephalus associated with intracranial schwannomas: hyperproteinorrachia as an etiopathological factor? Acta Neurochir (Wien) 2003;145:73-78.

7 Ashworth B, Gordon A: Leptomeningeal gliomatosis. J Neurol Neurosurg Psychiatry 1994;57:471-473.

8 Chang CC, Kuwana N, Ito S, Koike Y, Kitamura H: Spinal leptomeningeal metastases of giant cell glioblastoma associated with subarachnoid haemorrhage: case report. J Clin Neurosci 2001;8:56-59. 